\title{
Systematic construction of square-root topological insulators and superconductors
}

\author{
Motohiko Ezawa (ㅇ \\ Department of Applied Physics, University of Tokyo, Hongo 7-3-1, 113-8656, Japan
}

(Received 26 May 2020; accepted 19 August 2020; published 11 September 2020)

\begin{abstract}
We propose a general scheme to construct a Hamiltonian $H_{\text {root }}$ describing a square root of an original Hamiltonian $H_{\text {original }}$ based on the graph theory. The square-root Hamiltonian is defined on the subdivided graph of the original graph of $H_{\text {original }}$, where the subdivided graph is obtained by putting one vertex on each link in the original graph. When $H_{\text {original }}$ describes a topological system, there emerge in-gap edge states at nonzero energy in the spectrum of $H_{\text {root }}$, which are the inherence of the topological edge states at zero energy in $H_{\text {original }}$. In this case, $H_{\text {root }}$ describes a square-root topological insulator or superconductor. Typical examples are square roots of the Su-Schrieffer-Heeger (SSH) model, the Kitaev topological superconductor model, and the Haldane model. Our scheme is also applicable to non-Hermitian topological systems, where we study an example of a nonreciprocal non-Hermitian SSH model.
\end{abstract}

DOI: 10.1103/PhysRevResearch.2.033397

\section{INTRODUCTION}

Topological insulators and superconductors are among the most studied fields in condensed matter physics in this decade $[1,2]$. They are characterized by the emergence of topological edge states although the bulk is gapped.

They may have various extensions. Higher order topological insulators are one of them [3-13]. The higher order topological insulator looks trivial since it has no zero-energy edge states. However, there emerge zero-energy corner states isolated both from the edge band and the bulk band due to the nontrivial topology of the bulk. When we are ignorant of this fact, the emergence of zero-energy corner states looks accidental. However, as we have just noted, a deeper analysis has revealed that corner states emerge because the bulk is topological. This finding implies that some systems previously thought to be a trivial insulator can be a topological insulator by generalizing the concept of topology.

Recently, square-root topological insulators were proposed $[14,15]$. Its notion has been generalized to square-root higher order topological insulators [16]. They are characterized by the emergence of in-gap edge states clearly isolated from the bulk band. At first sight, its emergence looks accidental since they emerge at nonzero energy. However, there is a case whose origin can be traced back to the nontrivial topology of a closely related system, i.e., the nontrivial topology of its squared Hamiltonian. It is rather surprising that certain in-gap edge states can be brought to zero-energy topological edge states by squaring the Hamiltonian.

Published by the American Physical Society under the terms of the Creative Commons Attribution 4.0 International license. Further distribution of this work must maintain attribution to the author(s) and the published article's title, journal citation, and DOI.
In this paper, we propose a general scheme to construct square-root topological insulators and superconductors from ordinary topological insulators and superconductors based on the graph theory. There is one-to-one correspondence between a tight-binding Hamiltonian and a weighted graph. A graph is composed of vertices and links. We can construct a new graph by introducing one vertex on each link, which we refer to as the subdivided graph $[17,18]$. We call the original graph the parent graph in contrast to the subdivided graph. Any subdivided graph is bipartite because it contains original vertices and newly added vertices. Examples are shown in Figs. 1-3 in Sec. III, where original (new) vertices are shown in magenta (cyan).

We denote the Hamiltonian constructed on the subdivided graph as $H_{\text {root }}$. We then find $\left(H_{\text {root }}\right)^{2}=H_{\text {par }} \oplus H_{\text {res }}$, where $H_{\text {par }}$ is identical to the original Hamiltonian $H_{\text {original }}$ up to an additive constant interpreted as a self-energy. We call $H_{\text {par }}$ and $H_{\text {res }}$ the parent and residual Hamiltonians, respectively. When $H_{\text {original }}$ describes a topological system, it contains zero-energy topological edge states, producing the corresponding edge states at nonzero energy in $H_{\text {root }}$. Furthermore, zero-energy perfect-flat bulk bands may emerge in $H_{\text {root }}$ as a bipartite property according to the Lieb theorem: See orange lines in Figs. 2 and 3. Because the eigenvalues are shown to be identical between $H_{\text {par }}$ and $H_{\text {res }}$ except for zero-energy states in $H_{\text {res }}, H_{\text {root }}$ is interpreted as the square-root Hamiltonian of $H_{\text {original }}$. We can use the same topological index between $H_{\text {root }}$ and $H_{\text {original }}$ since the eigenvectors are identical between them. Indeed, the region of the in-gap edge states in $H_{\text {root }}$ is precisely the same as that of the zero-energy edge states in $H_{\text {original }}$. Namely, the phase diagram is identical between $H_{\text {root }}$ and $H_{\text {original }}$.

We present explicit examples of the Su-Schrieffer-Heeger (SSH) model, the Kitaev $p$-wave topological superconductor model, and the Haldane honeycomb model. Furthermore, our results are applicable to non-Hermitian systems, where we demonstrate an example of nonreciprocal non-Hermitian SSH model. 

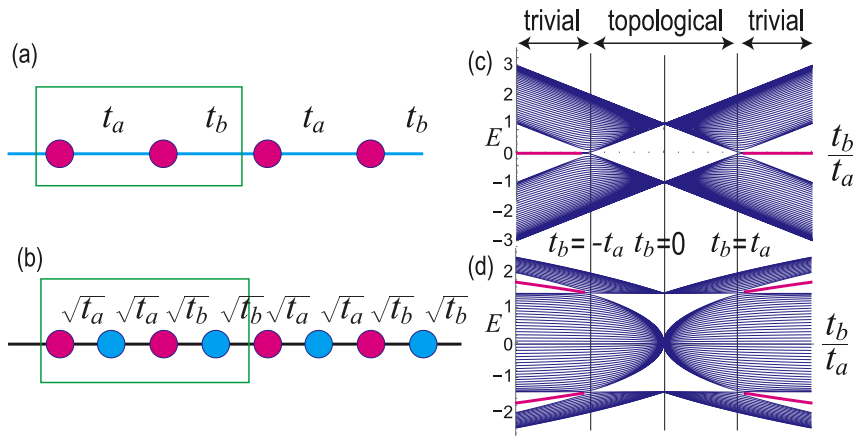

FIG. 1. Illustration of (a) the graph and (b) the subdivided graph for the SSH model $H_{\mathrm{SSH}}$. The green rectangles represent the unit cells. Energy spectrum of (c) $H_{\mathrm{SSH}}$ and (d) $H_{\text {root }}$ in unit of $t_{a}$ as a function of $t_{b} / t_{a}$. The topological edge states are marked in magenta. In-gap edge states are on the curve $E= \pm \sqrt{\left|t_{a}\right|+\left|t_{b}\right|}$ for $H_{\text {root }}$.

\section{SQUARE-ROOT HAMILTONIAN}

It is impossible to construct a local hopping model only by taking a square root of the Hamiltonian matrix. A simple example is given by the SSH model,

$$
H_{\mathrm{SSH}}=\left(\begin{array}{cc}
0 & t_{a}+t_{b} e^{-i k} \\
t_{a}+t_{b} e^{i k} & 0
\end{array}\right) .
$$

A square root of the model is given by

$$
\sqrt{H_{\mathrm{SSH}}}=\left(\begin{array}{cc}
0 & \frac{\sqrt{E(k)}}{t_{a}+t_{b} e^{-i k}} \\
\frac{t_{a}+t_{b} e^{-i k}}{\sqrt{E(k)}} & 0
\end{array}\right),
$$

with $E(k)=\sqrt{t_{a}^{2}+t_{b}^{2}+2 t_{a} t_{b} \cos k}$, as we derive in Appendix A. This is an infinite-range hopping model.

Here we recall the Dirac idea to take a square root of the Klein-Gordon equation. He obtained the Dirac equation by introducing a matrix degree of freedom. The Dirac equation has various intriguing properties such as chirality and the index theorem, which are absent in the Klein-Gordon equation.

We propose to take a square root of a Hamiltonian by increasing a matrix degree of freedom as follows: (1) We
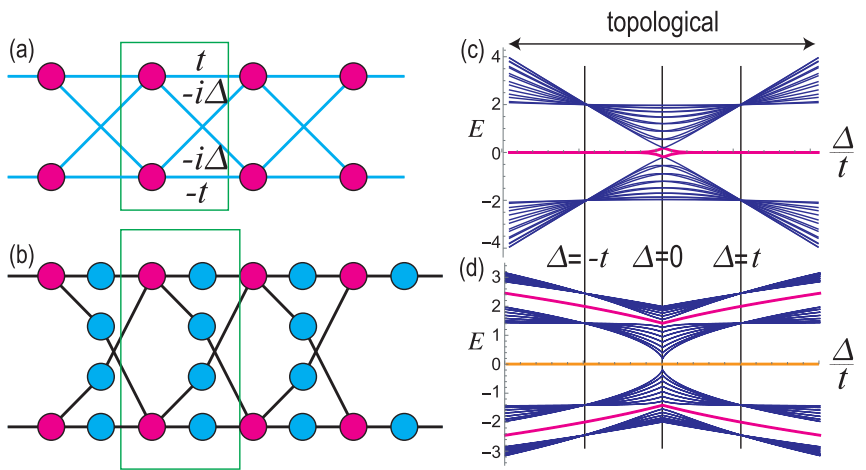

FIG. 2. Illustration of (a) the graph and (b) the subdivided graph for the Kitaev model $H_{\text {Kitaev }}$. Energy spectrum of (c) $H_{\text {Kitaev }}$ and (d) $H_{\text {root }}$ in unit of $t$ as a function of $\Delta / t$. The topological edge states are marked in magenta. In-gap states are on the curve $E=$ $\pm \sqrt{2|t|+2|\Delta|}$ for $H_{\text {root }}$. Lieb perfect-flat bulk-bands are marked in orange.
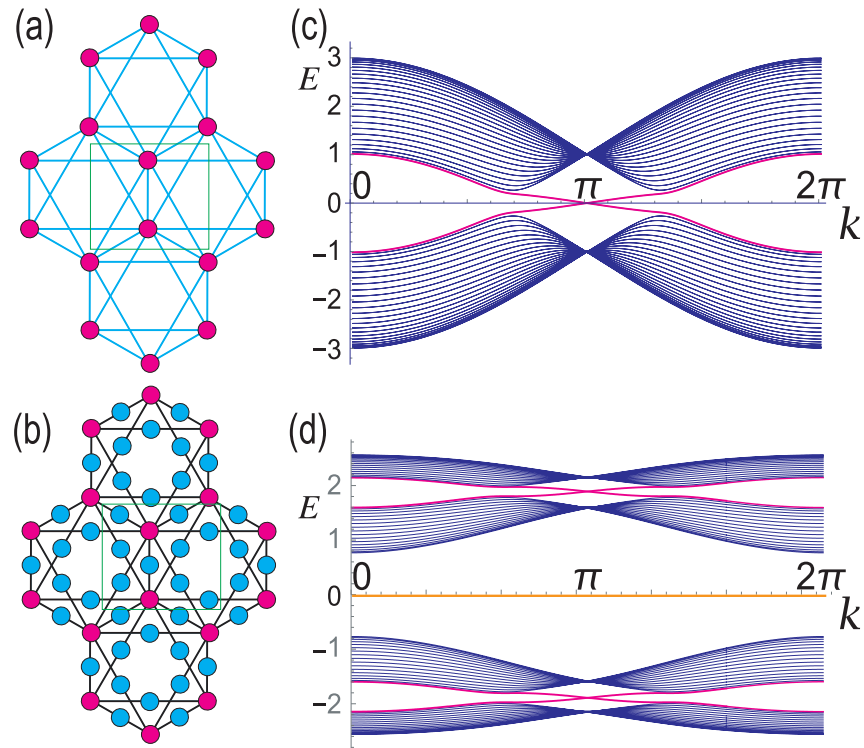

(d)

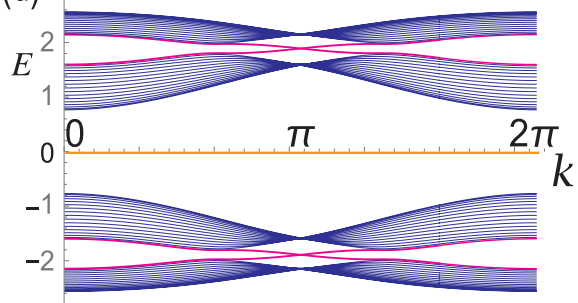

FIG. 3. Illustration of (a) the graph and (b) the subdivided graph for the Haldane model $H_{\text {Haldane }}$. Energy spectrum of (c) $H_{\text {Haldane }}$ and (d) $H_{\text {root }}$ in unit of $t$ as a function of the momentum $k$. The chiral edge states are marked in magenta. Lieb perfect-flat bulk bands are marked in orange. We have set $\lambda=0.2 t /(3 \sqrt{3})$.

first write down a graph representation of the adjacency matrix of the original Hamiltonian $H_{\text {original }}$. (2) We construct a subdivided graph from the original graph. (3) We construct a Hamiltonian $H_{\text {root }}$ on the subdivided graph. Then, we obtain $\left(H_{\text {root }}\right)^{2}=H_{\text {par }} \oplus H_{\text {res }}$, where $H_{\text {par }}$ is identical to the original Hamiltonian $H_{\text {original }}$ up to an additive constant, provided the hopping parameter is taken to be $\sqrt{t}$ in $H_{\text {root }}$ corresponding to the hopping parameter $t$ in $H_{\text {original }}$. The square-root Hamiltonian is given by $H_{\text {root }}$. When the hopping parameter $t$ is complex, we take simply a square root of it. For instance, when the hopping has a phase $e^{i \theta}|t|$, we set $e^{i \theta / 2}|t|^{1 / 2}$ for the square-root Hamiltonian.

We make a comment when the Hamiltonian has the spin or orbital degrees of freedom. Although a vertex of a graph cannot include internal indices, it is possible to introduce several vertices corresponding to internal indices. For example, we introduce two vertices to incorporate the spin degree of freedom.

We start with a Hamiltonian $H_{\text {original }}$ where a unit cell contains $N$ sites connected by $M$ hoppings. We consider a Hamiltonian on the subdivided graph, which is given by

$$
H_{\text {root }}=\left(\begin{array}{ll}
O_{N \times N} & H_{N \times M}^{\text {left }} \\
H_{M \times N}^{\text {right }} & O_{M \times M}
\end{array}\right) .
$$

It is required that $H_{M \times N}^{\text {right }}=\left(H_{N \times M}^{\text {left }}\right)^{\dagger}$, when $H_{\text {root }}$ is Hermitian. We have

$$
\left(H_{\text {root }}\right)^{2}=\left(\begin{array}{cc}
H_{\mathrm{par}} & 0 \\
0 & H_{\text {res }}
\end{array}\right)=H_{\mathrm{par}} \oplus H_{\mathrm{res}}
$$

where

$$
H_{\mathrm{par}} \equiv H_{N \times M}^{\mathrm{left}} H_{M \times N}^{\mathrm{right}}, \quad H_{\mathrm{res}} \equiv H_{M \times N}^{\mathrm{right}} H_{N \times M}^{\mathrm{left}} .
$$


Thus, the square of the Hamiltonian, $\left(H_{\text {root }}\right)^{2}$, is uniquely decomposed into a direct sum of $H_{\text {par }}$ and $H_{\text {res }}$, which are the parent and residual Hamiltonians defined on the parent and residual graphs. The unique decomposition of the square of a subdivided graph is understood as follows. We label A and $B$ vertices of the subdivided graph since it is bipartite. The square graph is grouped into two graphs, where one is composed only by the A vertices and the other is composed only by the B vertices. Thus, the decomposition of the graph is unique.

In general, $H_{\mathrm{par}}$ is identical to $H_{\text {original }}$ up to a constant term because both of them are constructed on the same graph,

$$
H_{\text {par }}=C+H_{\text {original }},
$$

where $C$ is a positive constant obtained by calculating $\left(H_{\text {root }}\right)^{2}$. This constant term can be interpreted as a self energy, as in the case of the second-order perturbation theory. The zero-energy topological edge states in $H_{\text {original }}$ are transformed into the ingap edge states at nonzero energy $\pm \sqrt{C}$ in $H_{\text {root }}$.

We show that it is enough to set the hopping $\sqrt{t}$ in the subdivided graph for $H_{\text {root }}$ in order to reproduce the parent Hamiltonian $H_{\text {par }}$ with the hopping $t$. We consider hoppings between three sites indexed by $i-1, i$, and $i+1$, where the sites $i-1$ and $i+1$ are nearest-neighbor sites in $H_{\text {original }}$ while the site $i$ is the additional site introduced in $H_{\text {root }}$. The relevant part of the Hamiltonian is explicitly given by

$$
H_{\text {root }}^{\prime}=t_{i}^{\text {left }} c_{i-1}^{\dagger} c_{i}+t_{i}^{\text {right }} c_{i}^{\dagger} c_{i-1}+t_{i+1}^{\text {left }} c_{i}^{\dagger} c_{i+1}+t_{i+1}^{\text {right }} c_{i+1}^{\dagger} c_{i},
$$

whose square is uniquely decomposed into a direct product,

$$
\left(H^{\prime}\right)^{2}=H_{\mathrm{par}}^{\prime} \oplus H_{\mathrm{res}}^{\prime},
$$

where

$$
\begin{aligned}
H_{\mathrm{par}}^{\prime} & =t_{i}^{\text {left }} t_{i}^{\text {right }} c_{i-1}^{\dagger} c_{i-1}+t_{i+1}^{\text {left }} t_{i+1}^{\text {right }} c_{i+1}^{\dagger} c_{i+1} \\
& +t_{i}^{\text {left }} t_{i+1}^{\text {left }} c_{i-1}^{\dagger} c_{i+1}+t_{i+1}^{\text {right }} t_{i}^{\text {right }} c_{i+1}^{\dagger} c_{i-1} .
\end{aligned}
$$

The hoppings are between the next-nearest-neighbor sites, and there are on-site potentials. It is necessary to set $t_{i}^{\text {left }}=t_{i}^{\text {right }}=$ $\sqrt{t}$ to obtain

$$
H_{\mathrm{par}}^{\prime}=t\left(c_{i-1}^{\dagger} c_{i-1}+c_{i+1}^{\dagger} c_{i+1}+c_{i-1}^{\dagger} c_{i+1}+c_{i+1}^{\dagger} c_{i-1}\right),
$$

which represents the generic terms in the original Hamiltonian $H_{\text {original }}$ defined on the subdivided graph. It shows that the Hamiltonian $H_{\text {root }}^{\prime}$ with the hopping amplitude $\sqrt{t}$ on the subdivided gives the parent Hamiltonian $H_{\text {par }}^{\prime}$ with the hopping $t$.

In this derivation, we find that it is impossible to tune the on-site potential independently. We have constructed a squareroot Hamiltonian only when the on-site terms are constant and equals to the hopping term $t$ by using the subdivided graph in general. In other words, it is impossible to construct a squareroot Hamiltonian if there are site-dependent on-site terms.

The bipartite Hamiltonian has chiral symmetry, $\left\{\gamma, H_{\text {root }}\right\}=0$, with the chiral operator defined by

$$
\gamma=\left(\begin{array}{cc}
I_{N \times N} & O_{N \times M} \\
O_{M \times N} & -I_{M \times M}
\end{array}\right) .
$$

In general, we have $M>N$. According to the Lieb theorem [19], there are $|M-N|$ zero-energy states constituting perfect-flat bulk bands.

It is known $[20,21]$ that the eigenvalues are identical between $H_{\text {par }}$ and $H_{\text {res }}$ except for these zero-energy states in $H_{\text {res }}$ and that they are non-negative. See also Appendix B. Namely, when we set

$$
H_{\mathrm{par}}\left|\psi_{j}^{\mathrm{par}}\right\rangle=\varepsilon_{j}\left|\psi_{j}^{\mathrm{par}}\right\rangle, \quad H_{\mathrm{res}}\left|\psi_{j}^{\mathrm{res}}\right\rangle=\varepsilon_{j}^{\mathrm{res}}\left|\psi_{j}^{\mathrm{res}}\right\rangle,
$$

we obtain $\left\{\varepsilon_{j}^{\text {res }}\right\}=\left\{\varepsilon_{1}, \ldots \varepsilon_{N}, 0, \ldots, 0\right\}$ and $\varepsilon_{j} \geqslant 0$. It follows from (6) that the eigenvectors of $H_{\text {par }}$ and $H_{\text {original }}$ are the same,

$$
H_{\text {original }}\left|\psi_{j}^{\mathrm{par}}\right\rangle=\left(\varepsilon_{j}-C\right)\left|\psi_{j}^{\mathrm{par}}\right\rangle .
$$

Furthermore, the eigenvectors $\left|\psi_{j}^{\text {res }}\right\rangle$ of $H_{\text {res }}$ are obtained from those of $H_{\mathrm{par}}$ as

$$
\left|\psi_{j}^{\mathrm{res}}\right\rangle \equiv \sum_{k}\left(H_{M \times N}^{\mathrm{right}}\right)_{j k}\left|\psi_{k}^{\mathrm{par}}\right\rangle
$$

as we derive in Appendix B.

When the Hamiltonian $H_{\text {root }}$ is diagonalized by a unitary transformation $U$ as $U^{\dagger} H_{\text {root }} U=H_{\text {root }}^{\mathrm{D}}$, the Hamiltonian $\left(H_{\text {root }}\right)^{2}$ is also diagonalized by the same unitary transformation as $U^{\dagger}\left(H_{\text {root }}\right)^{2} U=\left(H_{\text {root }}^{\mathrm{D}}\right)^{2}$. Then, the eigenvalues of $H_{\text {root }}$ are obtained just by taking a square root of them with the same eigenvectors,

$$
H_{\text {root }}\left|\psi_{j}^{\text {root }}\right\rangle=\varepsilon_{j}^{\text {root }}\left|\psi_{j}^{\text {root }}\right\rangle
$$

where

$$
\varepsilon_{j}^{\text {root }}=\left\{ \pm \sqrt{\varepsilon_{1}}, \ldots, \pm \sqrt{\varepsilon_{N}} ; \pm \sqrt{\varepsilon_{1}}, \ldots, \pm \sqrt{\varepsilon_{N}}, 0, \ldots, 0\right\} .
$$

Because of this property, $H_{\text {root }}$ is interpreted as the square-root Hamiltonian of $H_{\text {original }}$.

Consequently, non-zero-energy in-gap states emerge in $H_{\text {root }}$, which corresponds to the zero-energy states in $H_{\text {par }}$. As we shall see soon, the phase diagram which determined by the edge states is identical between $H_{\text {root }}$ and $H_{\text {par }}$. Hence, the same topological number is assigned for $H_{\text {root }}$ as that for $H_{\text {par }}$. See the instances of the phase diagrams of $H_{\text {par }}$ and $H_{\text {root }}$ in Figs. 1(c) and 1(d) for the SSH model and in Figs. 4(c) and 4(d) for the non-Hermitian model.

\section{EXAMPLES}

\section{A. Square-root SSH model}

For the first example, we analyze the SSH model (1). The spectrum contains the zero-energy topological edge states as in Fig. 1(c). The graph of the SSH model is a simple onedimensional graph containing two vertices in the unit cell [Fig. 1(a)]. The corresponding subdivided graph is a onedimensional graph containing four vertices in the unit cell [Fig. 1(b)]. The square-root Hamiltonian $H_{\text {root }}$ is given by (3) with $(N, M)=(2,2)$, and

$$
H_{2 \times 2}^{\text {left }}=\left(\begin{array}{cc}
\sqrt{t_{a}} & \sqrt{t_{b}} e^{-i k} \\
\sqrt{t_{a}} & \sqrt{t_{b}}
\end{array}\right) .
$$


(a)

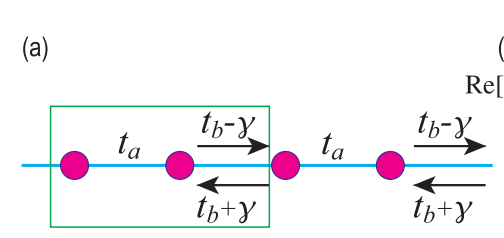

$\operatorname{Im}[E]$

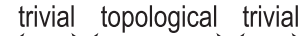

3

e $[E]$

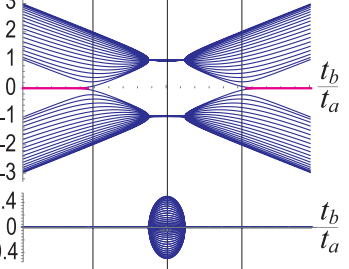

(b)

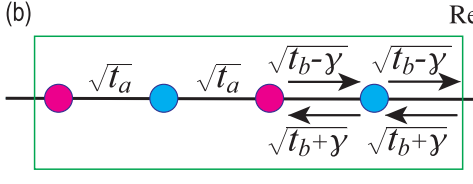

(d)
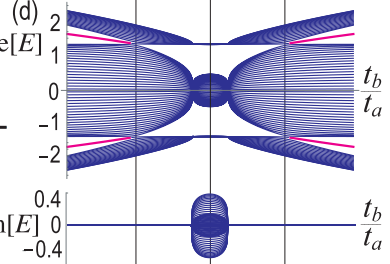

FIG. 4. Illustration of (a) the graph and (b) the subdivided graph for the nonreciprocal non-Hermitian SSH model $H_{\mathrm{SSH}}^{\text {non }}$. Real and imaginary parts of the energy spectrum of (c) $H_{\mathrm{SSH}}^{\text {non }}$ and (d) $H_{\text {root }}$ in unit of $t_{a}$ as a function of $t_{b} / t_{a}$. The topological edge states are marked in magenta. In-gap states are on the curve $E= \pm \sqrt{\left|t_{a}\right|+\sqrt{t_{b}^{2}-\gamma^{2}}}$ for $H_{\text {root }}$. We have set $\gamma=t_{a} / 4$.

It is straightforward to derive $\left(H_{\text {root }}\right)^{2}=H_{\text {par }} \oplus H_{\text {res }}$ with

$$
\begin{gathered}
H_{\mathrm{par}}=\left|t_{a}\right|+\left|t_{b}\right|+H_{\mathrm{SSH}}, \\
H_{\mathrm{res}}=\left(\begin{array}{cc}
2 t_{a} & \sqrt{t_{a} t_{b}}\left(1+e^{-i k}\right) \\
\sqrt{t_{a} t_{b}}\left(1+e^{i k}\right) & 2 t_{b}
\end{array}\right),
\end{gathered}
$$

where $H_{\text {res }}$ is the Rice-Mele model. In-gap edge states appear at $E= \pm \sqrt{\left|t_{a}\right|+\left|t_{b}\right|}$ for $\left|t_{b}\right|>\left|t_{a}\right|$, as illustrated in Fig. 1(d), whose origin is the topological zero-energy states in the SSH model [Fig. 1(c)]. The phase diagram of the square-root SSH model is found to be identical to that of the SSH model.

\section{B. Square-root Kitaev topological superconductor}

The next example is a square root of the Kitaev p-wave topological superconductor model defined by [22-25]

$$
H_{\text {Kitaev }}=(2 t \cos k-\mu) \sigma_{z}+2 \Delta \sigma_{x} \sin k .
$$

The spectrum contains the zero-energy topological edge states as in Fig. 2(c). The corresponding graph and subdivided graph are shown in Figs. 2(a) and 2(b). The square-root Hamiltonian $H_{\text {root }}$ is given the Hamiltonian (3) with $(N, M)=(2,4)$, and

$H_{2 \times 4}^{\text {left }}=\left(\begin{array}{cccc}\sqrt{t}\left(1+e^{-i k}\right) & 0 & \Delta^{\prime} & \Delta^{\prime *} e^{-i k} \\ 0 & i \sqrt{t}\left(1-e^{-i k}\right) & \Delta^{\prime *} e^{-i k} & \Delta^{\prime}\end{array}\right)$,

where $\Delta^{\prime}=e^{-i \pi / 4} \sqrt{\Delta}$.

We calculate $\left(H_{\text {root }}\right)^{2}=H_{\text {par }} \oplus H_{\text {res }}$. The parent Hamiltonian $H_{\mathrm{par}}$ is found to be the Kitaev Hamiltonian [22-25] with $\mu=0$ and the addition of a constant term $2|t|+2|\Delta|$. Ingap edge states appear in $H_{\text {root }}$ at $E= \pm \sqrt{2|t|+2|\Delta|}$ as in Fig. 2(d), which are transformed from the zero-energy topological states in the Kitaev model [Fig. 2(c)]. Furthermore, there are perfect-flat bulk bands at zero energy in $H_{\text {root }}$ due to the Lieb theorem [19] with $|M-N|=2$.

\section{Square-root Haldane model}

We next study a square root of the Haldane model. The Hamiltonian is defined on the graph in Fig. 3(a) and given by

$$
H_{\text {Haldane }}=2 \lambda\left(2 \sin \frac{k_{x}}{2} \cos \frac{\sqrt{3} k_{y}}{2}-\sin k_{x}\right) \sigma_{z}+t\left(1+\cos \frac{\sqrt{3} k_{x}}{2} \cos \frac{k_{y}}{2}\right) \sigma_{x}+t\left(\cos \frac{\sqrt{3} k_{x}}{2} \sin \frac{k_{y}}{2}\right) \sigma_{y} .
$$

The spectrum in nanoribbon geometry contains chiral edge states as in Fig. 3(c). The subdivided graph of the honeycomb graph is shown in Fig. 3(b). The square-root Hamiltonian $H_{\text {root }}$ is given by the Hamiltonian (3) with $(N, M)=(2$, 9), where

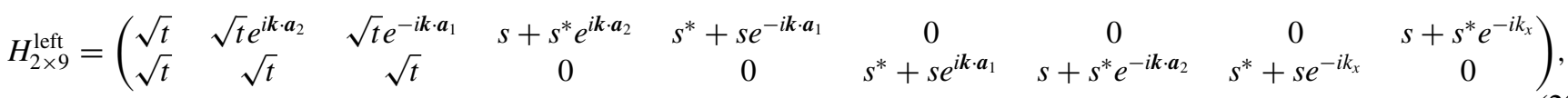

with $s=e^{i \pi / 4} \sqrt{\lambda}, \boldsymbol{a}_{1}=\{\sqrt{3} / 2,1 / 2\}, \boldsymbol{a}_{2}=\{\sqrt{3} / 2,-1 / 2\}$. The parent Hamiltonian $H_{\mathrm{par}}$ is found to be

$$
H_{\text {par }}=3(|t|+2|\lambda|)+H_{\text {Haldane }} .
$$

The chiral edge state in nanoribbon geometry emerges in $H_{\text {root }}$, as shown in Fig. 3(d). Furthermore, there are seven zero energy states in $H_{\text {total }}$ due to the Lieb theorem [19] with $|M-N|=7$.

\section{Square-root non-Hermitian SSH model}

We proceed to construct a square root of a non-Hermitian SSH model by introducing the nonreciprocity $\gamma$, as illustrated in Fig. 4(a). The Hamiltonian reads [26-31]

$$
H_{\mathrm{SSH}}^{\mathrm{non}}=\left(\begin{array}{cc}
0 & t_{a}+\left(t_{b}+\gamma\right) e^{-i k} \\
t_{a}+\left(t_{b}-\gamma\right) e^{i k} & 0
\end{array}\right),
$$

where the hopping amplitudes are different between left and right goings. The spectrum contains zero-energy edges states in the topological phase, whose real and imaginary parts are shown in Figs. 4(c) and 4( $\left.\mathrm{c}^{\prime}\right)$. The square-root Hamiltonian $H_{\text {root }}$ is defined on the subdivided graph in Fig. 4(b), and given by the Hamiltonian (3) with

$$
\begin{aligned}
H_{2 \times 2}^{\text {left }} & =\left(\begin{array}{cc}
\sqrt{t_{a}} & \sqrt{t_{b}+\gamma} e^{-i k} \\
\sqrt{t_{a}} & \sqrt{t_{b}-\gamma}
\end{array}\right), \\
H_{2 \times 2}^{\text {right }} & =\left(\begin{array}{cc}
\sqrt{t_{a}} & \sqrt{t_{a}} \\
\sqrt{t_{b}-\gamma} e^{i k} & \sqrt{t_{b}+\gamma}
\end{array}\right) .
\end{aligned}
$$


The parent Hamiltonian $H_{\mathrm{par}}$ is found to be

$$
H_{\mathrm{par}}=\left|t_{a}\right|+\sqrt{t_{b}^{2}-\gamma^{2}}+H_{\mathrm{SSH}}^{\mathrm{non}} .
$$

The residual Hamiltonian is given by $H_{\text {res }}=\left\{a_{i j}\right\}$, where

$$
\begin{aligned}
& a_{11}=2 t_{a}, \quad a_{12}=\sqrt{t_{a}}\left(\sqrt{t_{b}-\gamma}+\sqrt{t_{b}+\gamma} e^{i k}\right), \\
& a_{21}=\sqrt{t_{a}}\left(\sqrt{t_{b}+\gamma}+\sqrt{t_{b}-\gamma} e^{i k}\right), \quad a_{22}=2 \sqrt{t_{b}^{2}-\gamma^{2}} .
\end{aligned}
$$

In-gap edge states emerge at $E= \pm \sqrt{\left|t_{a}\right|+\sqrt{t_{b}^{2}-\gamma^{2}}}$ for $\left|t_{a}\right|>\left|t_{b}\right|$ in $H_{\text {root, }}$ as shown in Fig. 4(d), which are transformed from the zero-energy topological edge states in $H_{\mathrm{SSH}}^{\text {non }}$. The phase diagram of the square-root non-Hermitian SSH model is found to be identical to that of the non-Hermitian SSH model.

The above model can be generalized as follows. In general, we obtain a square root of a non-Hermitian topological system by taking (3) with $H_{M \times N}^{\text {right }} \neq\left(H_{N \times M}^{\text {left }}\right)^{\dagger}$. For example, we take

$$
H_{2 \times 2}^{\text {left }}=\left(\begin{array}{cc}
t_{1}^{\text {left }} & t_{4}^{\text {left }} e^{-i k} \\
t_{2}^{\text {left }} & t_{3}^{\text {left }}
\end{array}\right), \quad H_{2 \times 2}^{\text {right }}=\left(\begin{array}{cc}
t_{1}^{\text {right }} & t_{2}^{\text {right }} \\
t_{4}^{\text {right }} e^{i k} & t_{3}^{\text {right }}
\end{array}\right) .
$$

By calculating $\left(H_{\text {root }}\right)^{2}=H_{\text {par }} \oplus H_{\text {res }}$, we obtain

$$
H_{\text {par }}=\left(\begin{array}{cc}
t_{1}^{\text {left }} t_{1}^{\text {right }}+t_{4}^{\text {left }} t_{4}^{\text {right }} & t_{1}^{\text {left }} t_{2}^{\text {right }}+t_{4}^{\text {left }} t_{3}^{\text {right }} e^{-i k} \\
t_{2}^{\text {left }} t_{1}^{\text {right }}+t_{3}^{\text {left }} t_{4}^{\text {right }} e^{i k} & t_{2}^{\text {left }} t_{2}^{\text {right }}+t_{3}^{\text {left }} t_{3}^{\text {right }}
\end{array}\right),
$$

which is nonreciprocal non-Hermitian in general.

\section{DISCUSSION}

We have presented a systematic method to construct square-root topological insulators and superconductors based on subdivided graphs. We recall that subdivided graphs naturally arise in electric circuits when we rewrite the Kirchhoff law in the form of the Schrödinger equation $[17,18]$. Hence, it would be natural to make experimental observation of squareroot topological systems with the use of electric circuits. We start with a lattice electric circuit. In the original graph, it contains voltage at the sites, which correspond to the vertices in the graph theory. We can define currents flowing between two adjacent sites, which corresponds to links in the graph theory. Both the in-gap nonzero-energy edge states and the zero-energy flat bands due to the Lieb theorem are to be observed by measuring impedance peaks [32-34]. Another possibility to realize square-root topological systems is a direct construction of lattice structures by photonic [15] or acoustic [35,36] systems.

\section{ACKNOWLEDGMENTS}

The author is very grateful to N. Nagaosa for helpful discussions on the subject. This work is supported by the Grants-in-Aid for Scientific Research from MEXT KAKENHI (Grants No. JP17K05490 and No. JP18H03676). This work is also supported by CREST, JST (No. JPMJCR16F1).

\section{APPENDIX A: NAIVE CONSTRUCTION}

We try to construct a square-root of a given Hamiltonian in a naive way, where we take a square root of a matrix representing the original Hamiltonian. First, we diagonalize the original Hamiltonian $H$ by a unitary transformation as

$$
U^{-1} H U=H_{\mathrm{D}},
$$

where

$$
H_{\mathrm{D}}=\operatorname{diag} .\left(\varepsilon_{1}, \ldots, \varepsilon_{N}\right)
$$

is a diagonal matrix whose components are eigenvalues $\varepsilon_{j}$ with $1 \leqslant j \leqslant N$. Here, $N$ is the dimension of the matrix $H$ and $H_{\mathrm{D}}$. Then a square-root Hamiltonian $\sqrt{H}$ is given by

$$
\sqrt{H}=U \sqrt{H_{\mathrm{D}}} U^{-1},
$$

where

$$
\sqrt{H_{\mathrm{D}}}=\operatorname{diag} .\left(\sqrt{\varepsilon_{1}}, \ldots, \sqrt{\varepsilon_{N}}\right) .
$$

A problem is that a square-root Hamiltonian $\sqrt{H}$ is an infinite-range hopping model even when we start with a local hopping model $H$. We see it for an example of the square root of the SSH model (1), or

$$
H_{\mathrm{SSH}}=\left(\begin{array}{cc}
0 & t_{a}+t_{b} e^{-i k} \\
t_{a}+t_{b} e^{i k} & 0
\end{array}\right) \text {. }
$$

It is diagonalize as

$$
H_{\mathrm{D}}=E(k) \sigma_{z}
$$

with an energy

$$
E(k)=\sqrt{t_{a}^{2}+t_{b}^{2}+2 t_{a} t_{b} \cos k},
$$

and a unitary matrix

$$
U=\frac{1}{\sqrt{2}}\left(\begin{array}{cc}
\frac{E(k)}{t_{a}+t_{b} e^{-i k}} & \frac{-E(k)}{t_{a}+t_{b} e^{-i k}} \\
1 & 1
\end{array}\right) .
$$

Then the square-root Hamiltonian is given by

$$
\sqrt{H}=\left(\begin{array}{cc}
0 & \frac{\sqrt{E(k)}}{t_{a}+t_{b} e^{-i k}} \\
\frac{t_{a}+t_{b} e^{-i k}}{\sqrt{E(k)}} & 0
\end{array}\right),
$$

which is an infinite-range hopping model.

\section{APPENDIX B: BIPARTITE GRAPH}

We have constructed the Hamiltonian $H_{\text {root }}$ on the subdivided graph and decomposed it as $\left(H_{\text {root }}\right)^{2}=H_{\text {par }} \oplus H_{\text {res }}$. The eigenvalues of $H_{\mathrm{par}}$ and $H_{\text {res }}$ have the following two properties:

(1) All of the eigenvalues are identical between $\varepsilon_{\text {par }}=\varepsilon_{\text {res }}$ except for the zero energy.

In order to prove it, we analyze the eigenvalue problem,

$$
H_{\mathrm{par}} \psi_{\mathrm{par}}=\lambda \psi_{\mathrm{par}},
$$

with $\lambda \neq 0$. By inserting $H_{\mathrm{par}}=H_{N \times M}^{\text {left }} H_{M \times N}^{\text {right }}$, we rewrite it as

$$
H_{N \times M}^{\text {left }} H_{M \times N}^{\text {right }} \psi_{\text {par }}=\lambda \psi_{\text {par }} .
$$

By multiplying $H_{M \times N}^{\text {right }}$ from the left hand, we obtain

$$
H_{M \times N}^{\text {right }} H_{N \times M}^{\text {left }} H_{M \times N}^{\text {right }} \psi_{\text {par }}=\lambda H_{M \times N}^{\text {right }} \psi_{\text {par }} .
$$


We now define

$$
\psi_{\text {res }} \equiv H_{M \times N}^{\text {right }} \psi_{\text {par }},
$$

and use $H_{\text {res }} \equiv H_{M \times N}^{\text {right }} H_{N \times M}^{\text {left }}$ to obtain from (B3) as

$$
H_{\text {res }} \psi_{\text {res }}=\lambda \psi_{\text {res }} \text {. }
$$

Hence, the eigenvalues of $H_{\text {par }}$ and $H_{\text {res }}$ are identical except for the zero energy.

(2) The eigenvalues for $H_{\text {par }}$ and $H_{\text {res }}$ are positive $\varepsilon_{\text {par }} \geqslant 0$ and $\varepsilon_{\text {res }} \geqslant 0$, when they are Hermitian.

In order to prove it, we note that

$$
H_{\mathrm{par}}=\left(H_{M \times N}^{\text {right }}\right)^{\dagger} H_{M \times N}^{\text {right }},
$$

$$
H_{\mathrm{res}}=\left(H_{N \times M}^{\mathrm{left}}\right)^{\dagger} H_{N \times M}^{\mathrm{left}},
$$

when they are Hermitian. For an $N$-dimensional vector $\psi_{N}$ and an $M$-dimensional vector $\psi_{M}$, we find

$$
\begin{aligned}
\left\langle\psi_{N}, H_{\mathrm{par}} \psi_{N}\right\rangle & =\left\langle H_{M \times N}^{\mathrm{right}} \psi_{N}, H_{M \times N}^{\mathrm{right}} \psi_{N}\right\rangle \\
& =\left|H_{M \times N}^{\mathrm{right}} \psi_{N}\right|^{2} \geqslant 0, \\
\left\langle\psi_{M}, H_{\mathrm{res}} \psi_{M}\right\rangle & =\left\langle H_{N \times M}^{\mathrm{left}} \psi_{M}, H_{N \times M}^{\mathrm{left}} \psi_{M}\right\rangle \\
& =\left|H_{N \times M}^{\mathrm{left}} \psi_{M}\right|^{2} \geqslant 0 .
\end{aligned}
$$

Hence, we have proven $\varepsilon_{\text {par }} \geqslant 0$ and $\varepsilon_{\text {res }} \geqslant 0$.
[1] M. Z. Hasan and C. L. Kane, Rev. Mod. Phys. 82, 3045 (2010).

[2] X.-L. Qi and S.-C. Zhang, Rev. Mod. Phys. 83, 1057 (2011).

[3] F. Zhang, C. L. Kane, and E. J. Mele, Phys. Rev. Lett. 110, 046404 (2013).

[4] W. A. Benalcazar, B. A. Bernevig, and T. L. Hughes, Science 357, 61 (2017).

[5] F. Schindler, A. M. Cook, M. G. Vergniory, Z. Wang, S. S. P. Parkin, B. A. Bernevig, and T. Neupert, Sci. Adv. 4, eaat0346 (2018).

[6] Y. Peng, Y. Bao, and F. von Oppen, Phys. Rev. B 95, 235143 (2017).

[7] J. Langbehn, Y. Peng, L. Trifunovic, F. von Oppen, and P. W. Brouwer, Phys. Rev. Lett. 119, 246401 (2017).

[8] Z. Song, Z. Fang, and C. Fang, Phys. Rev. Lett. 119, 246402 (2017).

[9] W. A. Benalcazar, B. A. Bernevig, and T. L. Hughes, Phys. Rev. B 96, 245115 (2017).

[10] C. Fang and L. Fu, Sci. Adv. 5, eaat2374 (2019).

[11] M. Ezawa, Phys. Rev. Lett. 120, 026801 (2018).

[12] M. Ezawa, Phys. Rev. B 98, 045125 (2018).

[13] M. Geier, L. Trifunovic, M. Hoskam, and P. W. Brouwer, Phys. Rev. B 97, 205135 (2018).

[14] J. Arkinstall, M. H. Teimourpour, L. Feng, R. El-Ganainy, and H. Schomerus, Phys. Rev. B 95, 165109 (2017).

[15] M. Kremer, I. Petrides, E. Meyer, M. Heinrich, O. Zilberberg, and A. Szameit, Nat. Commun. 11, 907 (2020).

[16] T. Mizoguchi, Y. Kuno, and Y. Hatsugai, arXiv:2004.03235.

[17] M. Ezawa, Phys. Rev. B 100, 165419 (2019).

[18] M. Ezawa, Phys. Rev. Res. 2, 023278 (2020).
[19] E. H. Lieb, Phys. Rev. Lett. 62, 1201 (1989).

[20] C. Wu, D. Bergman, L. Balents, and S. Das Sarma, Phys. Rev. Lett. 99, 070401 (2007).

[21] M. E. Zhitomirsky and H. Tsunetsugu, Phys. Rev. B 70, 100403(R) (2004).

[22] A. Y. Kitaev, Sov. Phys. Usp. 44, 131 (2001).

[23] J. Alicea, Rep. Prog. Phys. 75, 076501 (2012).

[24] M. Leijnse and K. Flensberg, Semicond. Sci. Technol. 27, 124003 (2012).

[25] C. W. J. Beenakker, Annu. Rev. Condens. Matter Phys. 4, 113 (2013).

[26] H. Schomerus, Opt. Lett. 38, 1912 (2013).

[27] S. Lieu, Phys. Rev. B 97, 045106 (2018).

[28] T. E. Lee, Phys. Rev. Lett. 116, 133903 (2016).

[29] C. Yin, H. Jiang, L. Li, R. Lu, and S. Chen, Phys. Rev. A 97, 052115 (2018).

[30] S. Yao and Z. Wang, Phys. Rev. Lett. 121, 086803 (2018).

[31] M. Ezawa, Phys. Rev. B 99, 201411(R) (2019).

[32] S. Imhof, C. Berger, F. Bayer, J. Brehm, L. Molenkamp, T. Kiessling, F. Schindler, C. H. Lee, M. Greiter, T. Neupert, and R. Thomale, Nat. Phys. 14, 925 (2018).

[33] C. H. Lee, S. Imhof, C. Berger, F. Bayer, J. Brehm, L. W. Molenkamp, T. Kiessling, and R. Thomale, Commun. Phys. 1, 39 (2018).

[34] M. Ezawa, Phys. Rev. B 98, 201402(R) (2018).

[35] H. Xue, Y. Yang, F. Gao, Y. Chong, and B. Zhang, Nat. Mater. 18, 108 (2019).

[36] X. Ni, M. Weiner, A. Alu, and A. B. Khanikaev, Nat. Mater. 18, 113 (2019). 